\title{
Les réseaux de petites entreprises sur les territoires : des objets méconnus
}

Paulette Pommier

\section{OpenEdition}

1 Journals

Édition électronique

URL : http://journals.openedition.org/communicationorganisation/2946

DOI : 10.4000/communicationorganisation.2946

ISSN : 1775-3546

Éditeur

Presses universitaires de Bordeaux

Édition imprimée

Date de publication : 2 octobre 2004

ISSN : 1168-5549

Référence électronique

Paulette Pommier, "Les réseaux de petites entreprises sur les territoires : des objets méconnus », Communication et organisation [En ligne], 25 | 2004, mis en ligne le 27 mars 2012, consulté le 10 décembre 2020. URL : http://journals.openedition.org/communicationorganisation/2946 ; DOI : https://doi.org/10.4000/communicationorganisation.2946

Ce document a été généré automatiquement le 10 décembre 2020.

(c) Presses universitaires de Bordeaux 


\title{
Les réseaux de petites entreprises sur les territoires : des objets méconnus
}

\author{
Paulette Pommier
}

1 Une des particularités du comportement des patrons des petites entreprises de notre pays serait leur individualisme, un individualisme si marqué qu'il leur interdirait toutes formes de collaboration avec d'éventuels concurrents et leur ferait adopter des positions retranchées d'isolement professionnel. C'est une réalité quelque peu différente que nous avons observée à l'occasion du projet qu'il nous a été donné de conduire à la DATAR au cours des cinq dernières années sur les réseaux d'entreprises, appelés après quelques universitaires, «systèmes productifs locaux $»^{1}$.

2 L'appréciation que nous portons aujourd'hui sur la capacité à coopérer de ces chefs d'entreprises n'est pas aussi définitive et tranchée que celle qui nous avait été décrite alors. À un moment où la production devient de plus en plus immatérielle et l'industrie de moins en moins visible dans l'économie nationale, nous voyons dans l'ouverture à leur environnement de ces chefs d'entreprise un motif de réconfort.

3 Après une présentation du contexte dans lequel la délégation à l'aménagement $d u$ territoire s'est intéressée au sujet des réseaux de petites entreprises, nous proposerons une analyse des ressorts et des moteurs de ces coopérations pour terminer par un aperçu de leurs effets sur les territoires qui se «maillent » de l'intérieur et entre eux, formant une mosaïque complexe.

\section{L'aménagement du territoire et l'économie des réseaux}

4 Après avoir considéré le territoire comme un espace inerte sur lequel pouvaient être installées, avec une efficacité jugée à peu près équivalente, les entreprises, la politique publique d'aménagement du territoire a sensiblement évolué, à partir de la fin des années 1980 vers une conception rénovée du territoire. Aux instruments classiques (subventions pour des transferts d'entreprises de la région la plus attractive, la région Ile de France, vers des régions plus défavorisées et subventions aux nouvelles implantations dans ces 
mêmes régions en retard de développement) sont venus s'adjoindre de nouveaux outils qui traduisent une perception différente des ressorts du développement économique et de la place du territoire dans ce développement.

Peu à peu la DATAR s'est rangée à l'idée que la dynamique des acteurs d'un territoire et la façon dont ils conjuguaient leurs compétences et leurs efforts comptaient autant et souvent plus pour une activité économique que sa stricte localisation géographique.

Les travaux de chercheurs, économistes et sociologues, sur les districts industriels italiens qui ont débuté en Italie avec A. Bagnasco en 1977 et Giacomo Becattini pour ne citer que les premiers, n'ont en effet que très tardivement trouvé un écho dans la sphère des décideurs publics français. Ce sujet (la production de biens industriels par des myriades de petites entreprises liées entre elles par des solidarités historiques et une division fonctionnelle des tâches) a longtemps été considéré comme une particularité de la Troisième Italie (Italie du Nord et du Centre). Les résultats économiques de ces systèmes économiques très localisés, appelés par Becattini, districts industriels, qui leur ont permis de passer de régions d'émigration, faiblement développées voire pauvres à des régions comptant parmi les plus dynamiques d'Europe n'ont guère davantage éveillé l'attention de nos décideurs politiques.

7 Pour eux, la France n'avait pas de raison de se reconnaitre dans l'histoire de ces communautés locales, blotties dans de petites vallées dont aucun grand nom de firme n'émergeait, tout occupée qu'elle était dans l'après-guerre, au cours des «Trente Glorieuses ", à se doter d'une puissante économie basée sur des champions nationaux promis à devenir des champions mondiaux. La période d'étatisation qui a suivi, dans les années 1980, n'a guère été plus favorable à un intérêt pour l'économie transalpine. Et quand le succès est devenu patent, notamment à travers les volumes de productions exportées par ces districts, celui-ci a été mis sur le compte d'un archaïsme suspect et à terme condamné.

8 A cette époque, un trait était également tiré sur la réalité de nos propres «districts industriels ». Pourtant en France aussi, à Nogent, à Oyonnax, à Cholet, à Thiers, à Ste Sigolène.. des groupes de petites entreprises parvenaient à se développer en partageant des savoir-faire qu'elles cherchaient à faire évoluer afin de conserver leur position sur un marché qui s'ouvrait de plus en plus sur l'extérieur.

9 Ces réseaux de petites entreprises souvent familiales qui parvenaient à conjuguer concurrence locale et mise en commun de connaissances et d'opportunités commerciales et qui surtout portaient le même attachement à leur région, étaient devenus, pour reprendre l'expression de Jean Saglio, ${ }^{2}$ alors « invisibles ».

10 L'analyse de ce sociologue aide à comprendre le retard français dans la prise en considération des systèmes productifs localisés puis dans la mise en place de politiques publiques en leur faveur, et ce, malgré des capacités d'adaptation et d'évolution dont un certain nombre a fait la preuve. Dans la Vallée de l'Arve, par exemple où plusieurs centaines de petits entrepreneurs sont passés maîtres dans l'art du décolletage du métal et qui a fait de cette vallée l'endroit où les grands "mécaniciens" viennent s'approvisionner. "Ces systèmes ont longtemps été considérés comme sans importance ». Entre autres raisons, souligne Jean Saglio, il y a le fait que les patrons des PME de ces tissus sont des "êtres ordinaires"; ils ne sont pas des représentants de la grande bourgeoisie. Ils partagent avec le groupe auquel ils sont intégrés une certaine idée de la solidarité locale. C'est le groupe qui en cas de difficultés ou de conflit trouve des solutions 
et invente des règles. Ces solutions ne viennent pas de l'État. Elles contribuent à la formation d'une identité collective.

11 Jusque dans les années 90, plusieurs phénomènes expliquent donc l'absence d'attention aux dynamiques industrielles, passées ou présentes, spécifiques à des territoires et reposant sur l'organisation de producteurs locaux. À compter de cette période, l'attitude de l'État va se modifier sensiblement, les effets de la mondialisation et sa moindre capacité à intervenir dans l'économie l'y incitant fortement. Il ne lui est plus guère possible d'être un investisseur majeur participant à la formation de grands groupes, ni même de subventionner significativement tel projet d'implantation ou de développement. D'ailleurs la Direction de la concurrence, la plus puissante de la Commission Européenne veille ! Privé de ces moyens d'action, l'État va devoir s'intéresser davantage aux facteurs dits "endogènes » du développement. Considérer l'économie à partir des territoires va exiger de sa part un changement de posture puisqu'il s'agit désormais moins de s'intéresser à de grands projets qu'à des dynamiques portées par des acteurs locaux. Les groupes d'entrepreneurs, les réseaux d'acteurs locaux vont peu à peu (re)trouver droit de cité. L'apport conceptuel des chercheurs évoqués plus haut ${ }^{3}$ va trouver dans ce contexte une réelle utilité. Ils contribueront à faciliter la formulation du projet d'action publique qui se dessine au milieu des années 90 à la Datar. La panoplie des concepts marshaliens tels que "atmosphère ou climat industriel» ou encore « externalités positives » est remise à l'honneur. Elle servira à conforter la réflexion et l'action tant au niveau national que régional et local.

Lorsqu'elle décidera en 1997 de donner une impulsion aux coopérations interentreprises et d'aider les PME à s'organiser au niveau des territoires, c'est naturellement que la DATAR aura recours à la terminologie utilisée par les chercheurs. Les observateurs extérieurs verront alors dans le choix de ce vocabulaire jugé hermétique, une manifestation de l'incorrigible technocratie de l'État central. À "système productif localisé » puis « local » alors retenu est donnée la définition suivante :

«ensemble d'entreprises regroupées dans un espace de proximité autour d'une activité, voire d'une technologie,

- entretenant entre elles des relations plus ou moins intenses mais également avec le milieu environnant et en particulier avec les institutions qui servent d'appui au système productif (les lycées, instances de formation, organismes de veille, services aux entreprises). Ces relations ne sont pas seulement marchandes; elles peuvent être informelles et produirent un supplément de valeur, et bénéficiant d'une coordination et d'interfaces industrie-recherche ou formation qui doivent stimuler le système et le placer sur des voies d'innovation. $»^{4}$

Pour justifier cette politique, un éclairage particulier est porté sur les conditions de la concurrence au niveau mondial et sur l'impérieuse nécessité pour un pays tel que la France aux coûts de main d'œuvre relativement élevés, d'avoir recours à des modes de production flexibles et innovants. Ce qui suppose des échanges intenses, facilités par la proximité, afin d'essayer d'avoir un temps d'avance dans l'offre de produits et de services. « Dans une économie changeante où domine la compétition par le temps, l'apprentissage rapide est la seule garantie des survie. Ce ne sont plus les gros qui écrasent les petits mais les rapides qui écrasent les lents. L'écosystème relationnel à la fois instance interne et externe est devenu le facteur clé de la performance... Or le territoire compris comme un système social et non seulement spatial est à l'évidence un fournisseur privilégié de ces ressources relationnelles ${ }^{5}$ 
Ce principe, à savoir que l'essentiel des facteurs de performance économique est contenu dans les territoires où des synergies sont à l'œuvre, la Délégation à l'aménagement du territoire et à l'action régionale va le faire progressivement sien et le partager avec ses partenaires institutionnels dans les différents ministères ou institutions à compétences économiques.

\section{Des réseaux aux dimensions multiples}

17 Après avoir resitué le contexte dans lequel se situe notre observation, nous voudrions, dans cette deuxième partie, explorer quelques hypothèses sur la motivation des chefs d'entreprises qui s'associent et se trouvent engagés dans ces «nouvelles» (nouveauté toute relative comme on l'a vu) façons de produire.

Au préalable, portons un regard rapide sur les « réseaux » (ou systèmes productifs locaux) qui constituent la matière de notre observation.

Sans, loin s'en faut, concerner la totalité des entreprises qui se trouvent situées à proximité les unes des autres sur un territoire et qui pourraient développer des formes coopératives de travail, l'organisation en système productif local a, sous l'effet de la politique engagée qui a stimulé ou simplement éclairé des phénomènes existants, sensiblement progressé au cours des dernières années. Elle concerne aujourd'hui, selon nos estimations, 500000 salariés environ pour 18000 entreprises $^{6}$.

20 Quant au nombre d'entreprises qui participent à un projet collectif formalisé à travers une association, une société commune, un groupement d'intérêt économique, une société à responsabilité simplifiée,., il est nettement plus réduit. Le poids mesuré à travers les effectifs salariés de ces entreprises se situe aux alentours de 4000 .

21 Mais c'est aux raisons qui amènent ces chefs d'entreprises à travailler étroitement ensemble, à s'associer dans des projets ou des structures, qu'elles soient formelles ou informelles, que nous voudrions consacrer l'analyse qui suit.

L'hypothèse que nous avançons est que la démarche de coopération s'alimente à plusieurs sources; qu'elle recouvre plusieurs dimensions et que c'est la conjugaison d'aspirations multiples qui lui assurent une certaine pérennité. Nous chercherons à illustrer nos hypothèses de quelques exemples concrets.

\section{Les résultats économiques : le mobile dominant affiché}

23 La première motivation des entreprises est sans nul doute d'ordre économique. Encore qu'aux yeux d'entreprises ayant pris l'habitude de vivre repliées sur elles-mêmes et qui se vivent comme concurrentes, les avantages économiques d'une association n'apparaissent pas évidents. Comme souligné plus haut, concurrence et coopération sont perçues, dans les représentations dominantes, comme antinomiques. La hiérarchisation des motivations qui vont conduire à des collaborations est donc sujette à caution. Les animateurs qui tentent d'organiser un réseau d'entreprises le savent bien puisqu'ils vont essayer d'abord de faire naître de la confiance et de la convivialité entre ces entreprises.

Une fois l'obstacle culturel à la coopération levé, c'est la démonstration des avantages que procure le partage de certains coûts (formation de leurs salariés, mise aux normes 
environnementales, règlement de problèmes de sécurité, participation à plusieurs à des opérations de promotion, recherche en commun d'innovations, gestion partagée de machines, achats groupés de matières premières..) qui amènera l'adhésion des chefs d'entreprises et les décidera à s'engager plus avant sur des projets communs.

Le développement d'actions commerciales et promotionnelles communes est une démarche courante qui figure parmi les premières proposées pour rassembler un premier noyau d'entreprises. Les initiatives correspondantes vont de la forme la moins contraignante (annuaire de compétences qui sont présentes dans chaque entreprise du groupe, logo collectif, site Internet commun ou participation à plusieurs à des salons professionnels) à des engagements plus audacieux comme la prospection à l'étranger de clients ou la création d'une marque collective.

Un autre exemple de démarche coopérative est le groupement d'achat de matières premières ou d'approvisionnement. Le bénéfice retiré de ces coopérations est immédiatement perceptible. Ainsi, les quatre fabricants de moules pour les verreries de la vallée de la Bresle qui se sont regroupés pour négocier ensemble leurs achats de matières premières ont fait diminuer de 15 à $40 \%$ le coût de leur poste « achats ». D'autres projets de regroupements s'effectueront sur l'objectif de s'équiper de matériels de production dont elles feront un usage partagé.

Cette question des moyens de production compte particulièrement dans les réseaux de sous-traitants. Ici les donneurs d'ordres vont jouer un rôle éminent puisque après une organisation pyramidale, hiérarchique entre donneurs d'ordre et sous-traitants, c'est une organisation en réseau qui est désormais exigée, les constructeurs souhaitant que le nombre d'entreprises participant à la réalisation du produit final soit le plus limité possible. Cette nouvelle organisation concerne autant les fournisseurs de premier rang que les sous-traitants de $2 \mathrm{e}$ et $3 \mathrm{e}$ rang. Pour eux, la survie passe par ces formes d'association.

C'est ainsi qu'à Belfort en Franche-Comté, une trentaine de petites entreprises se sont associées pour créer Mecatem, société destinée à présenter à des donneurs d'ordres une offre complète en matière de conception et de réalisation de machines spéciales, dans le domaine de la mécanique, de l'usinage ou de la robotique.

Le processus de regroupement entre PME de ces secteurs qui fournissent des pièces et le plus souvent des éléments complexes et tout assemblés à de grands constructeurs n'est pas arrivé à son terme. Il devrait se poursuivre, ces constructeurs considérant que la mise en réseau est encore très insuffisante pour leur permettre d'être compétitifs par rapport à leurs concurrents étrangers. Le difficile challenge pour ces PME est de rester petites afin de conserver une bonne réactivité et souplesse d'adaptation et de se montrer aussi performantes que les grands sur le plan de l'innovation, de la réactivité et de la qualité.

Les entreprises sous-traitantes qui se trouvent sur le territoire du constructeur en perçoivent bien les attentes. À cette fin par exemple, la société Peugeot en Franche-Comté a encouragé la création de l'association Perfo-Est qui réunit une centaine de ses entreprises fournisseurs pour améliorer leur niveau de compétence. Mais nombre de sous-traitants se trouvent sur des territoires différents de celui du donneur d'ordre. Pour les entreprises de ces bassins secondaires de sous-traitance, les incitations publiques au regroupement vont s'avérer particulièrement utiles. La démarche de mise en réseau résultera en effet la plus part du temps d'une réflexion stratégique menée par des acteurs 
publics ayant des responsabilités à l'égard du territoire. C'est ce que nous avons observé avec deux SPL : Mecatronic en Val de Marne ou Mecanic Valley en Midi-Pyrénées.

\section{Du ludique à l'éthique, une autre gamme de motivations}

31 À ces motivations économiques viennent s'en adjoindre d'autres, de natures très différentes qui vont constituer à leur tour des moteurs plus ou moins puissants à la coopération. Elles vont changer quelque peu la nature des rapports des responsables d'entreprises entre eux, mais aussi leurs rapports avec les autres acteurs du territoire.

Ces motivations d'ordre non économique dont nous avons repéré quelques familles peuvent s'exercer simultanément. Elles se conjuguent parfois, le plus souvent même, et s'entrecroisent, voire se renforcent mutuellement.

Le plus visible de ce type de motivations est l'attachement au territoire, à son passé et à l'avenir qui s'y prépare. C'est le lien entre les générations, les passées et les actuelles, voire celles à venir qui constitue le ciment des projets communs. Les exemples abondent et la contemporanéité de la démarche sur les coopérations économiques (SPL) avec celle sur les territoires de projets (pays) à la fin des années 90 en a sans doute facilité l'expression. Si les acteurs économiques restent au cœur des coopérations, d'autres acteurs vont entrer dans le jeu et chercher à donner au projet un sens plus riche. Le tourisme et le projet culturel autour d'une économie du patrimoine se trouvent ainsi fréquemment associés à la démarche économique qui devient par ce biais l'affaire de la communauté locale. Les coopérations nées dans ces territoires à forte cohésion peuvent ainsi déboucher sur des projets de natures variées mais ils convergent tous sur une même ambition: le renforcement de la capacité du territoire à développer des emplois et à détendre ses entreprises.

Le pays de Tinchebray ou la Vallée des Alliages (tous deux en Basse-Normandie, Orne et Manche respectivement) en sont des exemples représentatifs. Ici les filières pèsent peu en termes d'emplois ou de valeur ajoutée. Mais leur valeur (historique et affective) comptent, elles, beaucoup.

À Tinchebray qui est une capitale historique de la quincaillerie puisqu'on y travaille le fer depuis l'époque gallo-romaine, la démarche fédérative a été encouragée par le projet de constitution du pays qui rassemble 138 communes. Réticentes car les rivalités étaient fortes, les entreprises se sont convaincues peu à peu de mutualiser leurs énergies et leurs compétences. Les premiers résultats ont porté sur la co-traitance de contrats et la mise en place de démarches commerciales collectives avec la participation de ces entreprises à plusieurs salons en Allemagne ou en Italie. Mais c'est l'état d'esprit de ces entreprises qui a surtout changé depuis que fonctionne le "pays». Le sentiment de solidarité est désormais bien ancré.

Forcalquier dans les Alpes de Haute-Provence, avec le SPL des saveurs et senteurs est un autre exemple de l'expression de solidarités enracinées dans un vieux pays bien circonscrit. Au centre du projet, se trouve le noyau « militant » qui, selon l'expression de ses promoteurs, est constitué des acteurs locaux décidés à s'engager personnellement pour le succès de la démarche. 

industriels italiens canoniques de G. Becattini, à la fois systèmes économiques et systèmes sociaux, à forte cohésion et dont on peut ici rappeler la définition : « une entité socioterritoriale caractérisée par la présence active d'une communauté de personnes et d'une population d'entreprises dans un espace géographique et historique donné » (Becattini 1992).

Ce sont ces réseaux qui seront les plus attentifs aux intrusions de groupes étrangers suspectés d'avoir des visées plus financières qu'économiques. Dans la Vallée de l'Arve (en Savoie), par exemple, la sensibilité aux prises de contrôles d'entreprises par des groupes ou des fonds de pension est très aiguë. Il ne s'agit pas comme à Fougères en 1935 avec le projet du député Etienne Le Poullen de fermer le territoire à la concurrence venue "d'étrangers " ». La mondialisation, les PME de la Vallée la pratiquent mais elles n'aiment guère que les plus beaux fleurons soient menacés de fermeture pour des raisons financières.

Une deuxième catégorie de motivation est celle que nous appellerons l'attachement à des «valeurs » hors marché. Nous en donnerons quelques illustrations. Pour le « cluster de la glisse » qui rassemble plus d'une centaine d'entreprises autour de l'activité du surf sur la côte basque autour de Biarritz, la valeur commune est la nature (la mer, la vague, la montagne). L'importance qui lui est donnée dépasse la prise en considération d'un élément naturel exceptionnel qui explique le succès de cette activité dans cette région. Cela va au delà puisque ici se développe une conception durable du développement signifiant de la part des entreprises la mise en application des principes de «responsabilité sociale des entreprises» (RSE). Celle-ci va amener les patrons des entreprises de ce tissu spécialisé ( $\mathrm{du}$ "shaper » à la plus grande) à défendre certes les paysages (dont les vagues) mais surtout à se poser en défenseur de l'environnement et des valeurs éthiques contenues dans le concept de développement durable, aussi bien dans ce lieu que dans ceux où les multinationales en particulier, (Oxbow, Quick...) exercent leurs activités. La défense de ces valeurs est favorisée par un comportement distinctif de la part des entrepreneurs, majoritairement surfeurs de haut niveau ${ }^{8}$

Une autre illustration est donnée par le SPL de l'agglomération de Grasse, autour du naturel (des essences naturelles par opposition aux essences artificielles, composants principaux des parfums actuels). Les acteurs et leaders de ce système qui sont sur des positions plus défensives que le précédent car Grasse n'est plus la capitale des parfums qu'elle fut au début du siècle passé, sont comme ceux de Biarritz, conscients d'être porteurs d'une image de consommation originale, voire à « contre-courant » (mais n'ontils pas l'intuition que ce qui

41 On notera les différents noms que se donnent les SPL qui sont de vrais noms de marque faits pour attirer et séduire les clients mais également pour fédérer les protagonistes de SPL en les incitant à porter haut ses « couleurs ». aujourd'hui est à contre-courant pourrait demain signifier une longueur d'avance ?).

«Silicon Sentier (SPL des TIC du centre de Paris) représente un autre exemple mais on pourrait tout aussi bien, sur la même filière, les TIC et les médias, évoquer " Lyon Game ${ }^{9}$ » (SPL des)eux vidéo à Lyon). Caractérisés par leur jeunesse, les patrons de ces entreprises qui sont absorbés par leur métier de développeur d'applications technologiques de pointe se trouvent localisés dans le cœur de villes, là où la perception d'une identité et la solidarité ave : un territoire est loin d'être évidente. Outre l'envie d'être les meilleurs sur 
leurs créneaux fortement concurrencés, ils partagent une certaine éthique. À Silicon Sentier, la soixantaine d'entreprises du noyau dur organise régulièrement des réunions avec les « citoyens utilisateurs » qui sont les habitants du quartier. Sur les six actions du programme que s'est fixé ce système productif local, l'une d'elles est la mise en place d'une «bourse du citoyen " sorte de bourse des compétences où les protagonistes échangent des connaissances en informatique contre des connaissances dans d'autres domaines : piano, littérature,...

Affirmant sa différence, ce réseau d'entreprises défend dans sa charte «un angle d'approche plus humain », qui cimente sa nouvelle identité collective au cœur de Paris.

La Cité de l'Initiative à Roubaix est un autre exemple de réseau d'entreprises qui s'est donné, dès l'origine, une dimension éthique et solidaire. Pour cela, ce réseau principalement constitué de façonniers de l'habillement a pris la forme d'une Union d'Economie Sociale, SARL sans but lucratif. Ses membres ont approuvé un règlement éthique par lequel ils s'engagent sur un certain nombre de principes tels que le respect du droit du travail, l'application d'une hiérarchie des salaires réduite, le privilège accordé lors d'embauches à des chômeurs de longue durée, handicapés ou femmes en recherche d'un premier emploi ou en difficulté sur le marché du travail..

Le pôle 16000 Image d'Angoulême s'est fixé pour sa part l'objectif d'apporter des services, notamment d'offrir des loisirs à bas prix, aux salariés des entreprises du pôle par le biais du Comité interentreprises des œuvres sociales.

Le SPL saveurs et senteurs des Alpes du Sud déjà cité a de la même façon cherché à " articuler une démarche économique et une démarche sociale ", plusieurs entreprises de ce SPL s'étant engagées dans un projet "citoyen» visant à faciliter l'intégration professionnelle de personnes non voyantes.

Enfin, on se référera à la volonté de certains réseaux de s'engager dans des opérations de co-développement avec des régions de pays du Sud (Ethiopie, Liban, Maghreb...). Sur des relations au départ centrées sur des objectifs économiques, s'est greffée la volonté de transmettre des savoir faire et de partager avec des acteurs de ces pays des expériences réussies.

Bien d'autres exemples où le "non économique » occupe une place dans le projet de coopération et la construction du réseau pourraient être donnés. Ce qui semble relier ces différentes expériences, c'est une volonté commune de s'engager dans de nouvelles aventures pour faire face aux défis posés par une économie très mondialisée et de s'en servir pour produire des œuvres collectives qui déboucheront sur plus de cohésion, de lien social, de sens. Il est trop tôt pour dire si à travers ces projets leurs auteurs parviennent à faire la démonstration que la compétitivité (territoriale) est intimement liée au degré de confiance que les acteurs entretiennent entre eux. En tout état de cause, ce qui frappe dans ces initiatives, c'est l'enthousiasme et la ferveur des personnes qui s'y engagent.

\section{Les réseaux : vers un espace économique et social de plus en plus maillé}

50 Ces coopérations, aux fondements divers, modifient peu à peu l'organisation de l'économie sur le territoire. Elles débouchent moins qu'on aurait pu le craindre sur une 
organisation cloisonnée, où les systèmes productifs locaux entretiendraient entre eux peu de liens. C'est ce point que nous souhaiterions aborder dans cette dernière partie.

Peut-être parce qu'en France leur renouveau ou leur apparition se situe dans une époque où la mondialisation-globalisation de l'économie est omniprésente, les organisations qui se développent ne sont pas restées repliées sur elles-mêmes. Très vite s'est développée en leur sein l'idée de partenariats inter organisations. Le sentiment de forte identité qu'aurait procuré une longue histoire industrielle et qu'une géographie pouvant confiner à l'enclavement serait venue conforter n'est pas en effet le lot des organisations productives françaises récentes. La dimension identitaire constitue, comme on l'a vu, une sorte de détour pour donner plus de sens à un projet qui ne veut pas se donner seulement une finalité économique. Aussi, les systèmes productifs locaux français ont-ils rapidement souhaité asseoir leur stratégie de réseau sur des partenariats avec des homologues plus ou moins éloignés. Le premier de ces réseaux à se constituer tut, à l'instigation de l'animatrice de la Cité de l'Initiative de Roubaix, celui opérant dans la filière textile. Etaitce le sentiment de vulnérabilité particulier à cette branche qui les poussait à se rapprocher? Ce qui est sûr c'est que très vite l'envie d'échanger selon une approche thématique s'est communiquée aux systèmes productifs locaux d'autres secteurs: TIC, médias, parfums et cosmétiques.

Cette mise en réseau des systèmes productifs locaux répondait à une première attente qu'expriment fréquemment les responsables du développement local: celui de ne pas rester isolé et d'apprendre au contact d'autres acteurs confrontés à des situations identiques. Une deuxième explication est venue du besoin de surmonter le handicap de la taille en se renforçant mutuellement. Ceci est particulièrement vrai dans la mécanique où très vite des complémentarités dans les productions ont été recherchées et trouvées. Elles constituent un encouragement à des rencontres fréquentes entre réseaux. Dans le textile, les associations entre réseaux ou entreprises n'ont encore que rarement débouché sur des productions partagées, les spécialités de chaque réseau étant très particulières: façonniers à Castres et Roubaix, textile technique dans le Nord, vêtement à Cholet, textile santé à Saint Etienne, broderie à Cambrai, dentelle à Calais.. Les rencontres entre animateurs ont pourtant eu lieu sans interruption depuis 1999 comme si ces échanges jouaient le rôle de «think tank » où chacun venait se ressourcer et puiser des idées pour le développement de son propre réseau.

Comme le souligne Régis Guillaume ${ }^{10}$ dans son étude des systèmes productifs locaux de Midi-Pyrénées, "au delà de leurs aspects très hétérogènes, l'un des enseignements majeurs des investigations conduites, est l'extrême ouverture de ces systèmes, à la fois sur leur environnement proche mais également vers des espaces beaucoup plus éloignés. Les liaisons entre les différents SPL existent et ne s'expriment pas exclusivement de façon hiérarchique. La construction de compétences transversales assises sur des réseaux relationnels illustre ce phénomène. L'intérêt que les entreprises des SPL de Mecanic Vallée ou de Metaladour par exemple retirent de ces liaisons est évident : se rapprocher géographiquement des donneurs d'ordres et s'inscrire parallèlement dans un processus de diversification qui pourra être utile au moment d'affronter les contraintes nouvelles imposées par ces mêmes donneurs d'ordres ».

Mais les réseaux débordent-ils des frontières nationales et les voit-on prendre une dimension internationale, à tout le moins européenne? 

pour créer des réseaux internationaux, nous convainc que le même maillage de réseaux productifs à l'échelle du continent européen se prépare et pourrait voir le jour dans peu de temps.

\section{Conclusion :}

61 En conclusion du panorama que nous venons de tracer, nous voudrions insister sur trois points :

Le premier a trait à l'importance et à la complexité du phénomène de réseau. Les moteurs de la coopération entre entreprises qui se construit sur des territoires donnés et dont la territorialité est une caractéristique essentielle sont d'abord économiques : il s'agit de 
produire plus et mieux en s'associant. L'intelligence collective est mise à profit pour développer des marchés, parfois de niche, stimuler la créativité, améliorer ses avantages comparatifs et donc produire de la différenciation et gagner en compétitivité. Mais en même temps, le fait d'être ensemble génère la recherche d'un autre contenu que chaque réseau se définit dans la sphère de l'éthique, de la cohésion sociale, du développement durable, de la solidarité,... Comme s'il était permis, parce qu'on est plus fort à plusieurs, de s'engager sur d'autres chemins et de construire une collaboration féconde entre ces deux dimensions du réseau. Mais sans doute faut-il comprendre que l'une a besoin de l'autre, que la dimension plaisir ou éthique renforce celle qui vise les résultats économiques.

Le second point porte sur les dimensions du réseau et de la coopération dont on sait qu'elles reposent en grande partie sur la personnalité de leaders parmi les patrons des entreprises ou les animateurs du réseau. Compte tenu de leur fonction, ces derniers savent prendre du recul, trouver les thèmes qui vont renforcer la cohésion du groupe, proposer des champs d'investissement qui vont permettre à la fois de consolider les relations interpersonnelles et constituer des occasions de se projeter dans d'autres univers avec sans doute, en retour, des bénéfices en matière d'innovation et de création. L'expérience a montré en effet que sans leaders ou animateurs charismatiques, les réseaux étaient plus lents à se constituer et bien moins résistants.

Enfin, nous voudrions insister sur l'intérêt qu'il y aurait à pousser plus loin l'analyse de ces objets si particuliers que sont les réseaux de production implantés dans les territoires afin de mieux les comprendre et sans doute de mieux les accompagner.

Nous souhaiterions vivement que ces « objets invisibles » ne le restent pas et que sur eux se focalisent davantage d'analyses approfondies selon des approches multidisciplinaires. Nous avons signalé combien les études d'historiens étaient utiles. D'autres disciplines (sociologie, économie, géographie) mériteraient d'être plus largement sollicitées qu'elles ne le sont aujourd'hui. Nous sommes convaincue que cette « nouvelle » façon de produire, à l'heure où tant de phénomènes économiques échappent à la maîtrise des gouvernements et des pouvoirs locaux, est utile au développement et au maintien de richesses mais elle l'est aussi pour ce qu'elle représente de valeurs et d'humanité.

\section{NOTES}

1. Pour une présentation de cette politique voir: Les systèmes productifs locaux DATAR, Documentation française- janvier 2002

2. «Des objets invisibles » Communication à paraître dans les Actes du Colloque 5-6 février 2004 - Mobilisation du territoire, les districts industriels en Europe occidentale du XVII ${ }^{\mathrm{e}}$ au XX ${ }^{\mathrm{e}}$ siècle. Comité pour l'histoire économique et financière de la France.

3. Une bibliographie de référente est donnée par Claude Courlel dam » Les systèmes productifs locaux: du concepì au modèle» dans Réseaux d'entreprises et territoires, DATAR, La documentation française - janvier 2001

4. Définition du cahier des charges de l'appel à projets lancé par la DATAR en 1998 et 1994. 
5. Des lieux el des liens, Politiques du territoire à l'heure de la mondialisation, Pierre Veltz Editions de l'Aube 2002.

6. Ce chiffre peut être mis en regard du nombre de salariés de l'industrie (3millions) et du nombre de ceux qui se trouvent appartenir à des "agglomérations industrielles » qui serait de l'ordre de 1,5 million. Voir pour une définition et une analyse des agglomérations d'entreprises : Michel Laine dans «Réseaux d'entreprises et territoires, Regards sur les systèmes produites locaux « - DATAR Documentation française -janvier 2001

7. Les ressorts du district fourgerais de la chaussure, XIX-XX ${ }^{\mathrm{e}}$ siècle, Florent le Bot, à paraître dans les Actes du colloque sur les districts industriels des 5 et 6 lévrier 2004.

8. Pour accéder à des postes de responsabilité dans tes entreprises, on nous a signalé qu'il fallait avoir gagné ses galons de surfeurs.

9. On noiera les différents noms que se donnent les SPL qui sont de vrais noms de marque faits pour attirer et séduire les clients mais également pour fédérer les protagonistes de SPL en les incitant à porter haut ses « couleurs "

10. Régis Guillaume (Cirus-Cieu (UT2) Maison de la recherche. Université de Toulouse Il I.e Mirail) «Les systèmes productifs locaux et gouvernance territoriale: vers l'émergence de systèmes régionaux »

11. Voir Etude sur districts italiens par la mission économique de l'Ambassade de France à Rome 2003

\section{RÉSUMÉS}

Il existerait dans les réseaux d'entreprises qui se sont constitués sur les territoires, dans les systèmes productifs locaux en particulier, une dimension cachée de la motivation de ses protagonistes. De nature non économique et à contenu souvent éthique, elle est apparue en dehors de tout modèle préétabli, à la différence de la dimension économique qui, elle, a été explicitement encouragée par les pouvoirs publics. Les patrons, à la tète de petites affaires seraient donc, lorsqu'ils font la démarche de conduire des projets communs, porteurs d'une conception du développement globalisante. Parce qu'il ne renvoie pas aux représentations traditionnelles de l'entrepreneur, ce comportement reste toutefois largement méconnu.

In the corporate networks which have been constituted on the territories, in the local productive systems in particular, a hidden dimension of the motivation of their protagonists is believed to exist. Of a non-economic nature and often with ethical contents, it appeared apart from any preestablished model, contrary to the economic dimension which was strongly encouraged by the authorities. The employers, responsible for small business units, would then, when they try to manage joint projects, have in mind a new concept of global development. This behaviour remains however largely ignored as it does not reflect the traditional image of the businessman. 


\section{AUTEUR}

\section{PAULETTE POMMIER}

Paulette Pommier est à la Délégation à l'Aménagement du territoire et à l'action régionale (DATAR) chargée du développement économique local et en particulier de l'encouragement aux formes particulières de coopération entre acteurs, notamment aux systèmes productifs locaux (SPL). Elle s'est spécialisée dans le thème du développement local et s'est attachée à en explorer les formes les plus participatives. Mail : paulete.pommier@datar.gouv.fr 